\title{
Effects of an intervention based on self-determination theory on self-reported leisure-time physical activity participation
}

\author{
Nikos L.D. Chatzisarantis ${ }^{\mathrm{a} *}$ and Martin S. Hagger ${ }^{\mathrm{b}}$ \\ ${ }^{a}$ School of Psychology, University of Plymouth, Plymouth, UK; ${ }^{b}$ School of Psychology, \\ University of Nottingham, Nottingham, UK
}

(Received 18 May 2006; final version received 12 November 2007)

\begin{abstract}
Based on self-determination theory, the present study developed and evaluated the utility a school-based intervention to change pupils' physical activity intentions and self-reported leisure-time physical activity behaviour. The study evaluated utility of the intervention to promote physical activity participation over a 5 -week interval of time. A cluster randomised design targeting 215 pupils from 10 schools with schools as the unit of randomisation was adopted $($ Male $=106$, Female $=109$, Age $=14.84, \mathrm{SD}=0.48)$. Results indicated that pupils who were taught by autonomy-supportive teachers reported stronger intentions to exercise during leisure time and participated more frequently in leisure-time physical activities than pupils in the control condition. Autonomous motivation and intentions mediated the effects of the intervention on self-reported physical activity behaviour. It is concluded that self-determination theory provides a useful framework for the development of school-based interventions that ultimately affect leisure-time physical activity participation.
\end{abstract}

Keywords: autonomy support; autonomous motivation; intentional processes

Self-determination theory is an influential theory of human motivation that explains a wide array of phenomena, such as motivation and psychological well-being, on the basis of a few principles related to three innate psychological needs for autonomy, competence and relatedness (Ryan \& Deci, 2000). Autonomy refers to the need to experience oneself as initiator and regulator of one's actions. Competence refers to the need to produce behavioural outcomes, and understand the instrumentalities leading to these behavioural outcomes. Relatedness refers to the need to experience satisfactory relationships with others and with the social order in general (Deci \& Ryan, 1985).

Although self-determination theory recognises the importance of psychological needs in understanding human motivation, it does not focus on variation in need strength. The critical issue is to investigate inter-personal variation in the satisfaction of basic needs, which is the result of being exposed to different need-supportive environments (Ryan \& Deci, 2000). Accordingly, self-determination theory differentiates between two types of environments. The environment is said to be autonomy supportive when the inter-personal style of people in positions of authority (i.e. head-teacher, parents) takes the perspective of

*Corresponding author. Email: nikos.chatzisarantis@plymouth.ac.uk 
individuals into consideration. Perspective taking satisfies need for relatedness and enhances a sense of belongingness (Deci, Eghrari, Patrick, \& Leone, 1994; Ryan \& Deci, 2000). In addition, environments are autonomy supportive when they explain in a meaningful way why performance of behaviour might be important. Provision of meaningful rationale can facilitate a sense of direction and enhance sense of competence when it is coupled with positive feedback (Ryan \& Deci, 2000). Finally, autonomysupportive environments satisfy need for autonomy by providing opportunities for choice and by using neutral language (e.g. use modal operators such as 'may' and 'could' rather than 'shoulds' and 'musts') during inter-personal communication (Deci et al., 1994). The environment is said to be controlling when two of the three critical factors making up autonomous environments (rationale, choice or perspective taking) are absent from the environment (Deci et al., 1994). For example, the environment is controlling when people in positions of authority do not provide meaningful rationale, use pressuring language during inter-personal communication (e.g. use of modal operators such as 'should' and 'must'), and/or when people in positions of authority pressure individuals accept their points of view (Deci et al., 1994).

Self-determination theory not only distinguishes between autonomous and controlling inter-personal styles and contexts but also between autonomous and controlling motivational styles. People who operate in autonomous contexts develop on average an autonomous motivational style and freely choose to adopt suggested behaviour because they find the behaviour intrinsically rewarding or because they perceive that the suggested behaviour is important and meaningful (Ryan \& Deci, 2000). In contrast, individuals operating in controlling contexts develop on average a controlling motivational style and tend to adopt suggested behaviour because they feel pressured or coerced to do so by significant others or because they feel that they should (Deci et al., 1994). Supporting this general set of assumptions, correlation studies have found relationships between autonomy support and autonomous motivational styles on the one hand, and between controlling inter-personal context and controlling motivational styles on the other hand (Ntoumanis, 2001; Reeve \& Jang, 2006; Standage, Duda, \& Ntoumanis, 2003, 2005; Williams, Gagné, Ryan, \& Deci, 2002). Further, there is evidence to suggest that motivational orientations partly mediate the effects of perceived autonomy support on actual and self-reported health behaviour (Hagger, Chatzisarantis, Barkoukis, Wang, \& Baranowski, 2005; Hagger, Chatzisarantis, Culverhouse, \& Biddle, 2003).

Although the sequence of motivated behaviour proposed by self-determination theory has been confirmed in several correlational studies, few intervention and experimental studies have tested self-determination theory in relation to health behaviours. In a schoolbased intervention programme, Vansteenkiste, Simons, Soenens and Lens (2004b) used tenets of self-determination theory to promote exercise participation (Taibo). Their intervention strategy involved short-persuasive messages that advocated benefits of Taibo in an autonomy supportive or controlling ways, and delivery of Taibo exercises by a certified instructor over two physical education classes. In accordance with tenets of selfdetermination theory, their study pointed out that autonomy-supportive environments enhanced pupils' perceptions of autonomy support, autonomous motivational orientations and actual persistence in Taibo classes. However, their intervention programme was brief producing small to medium effect sizes $\left(0.02<f^{2}<0.17\right)$ that explained between 2 and $21 \%$ of variance in exercise participation (Vansteenkiste, Simons, Lens, Sheldon, \& Deci, 2004a; Vansteenkiste, Simons, Lens, Soenens, \& Matos, 2005; Williams et al., 2002). In addition, Vansteenkiste et al. (2004b) study did not provide a rigorous test of utility of autonomy support in facilitating generality of behavioural change; that is transference of 
health behaviour from school settings to a leisure context (Deci \& Ryan, 1985). This is because their intervention programme offered Taibo classes in school settings and not outside of schools. The present study will provide a more rigorous test of generality of behavioural change by not offering exercise classes to young people after termination of the intervention.

Most critical, Vansteenkiste et al. (2004b) and other intervention studies (Williams et al., 2002) did not test previous laboratory findings suggesting that sense of choice and acknowledgement of difficulties are the most critical inter-personal factors facilitating autonomous motivation and persistence (Deci et al., 1994). This is because previous studies compared interventions that facilitated autonomy support against interventions that undermined sense of autonomy and choice (Williams et al., 2002). Choice and acknowledgement of difficulties was not varied across experimental and control conditions systematically. Overall, the present study extends previous research by (i) varying systematically choice and acknowledgement of difficulties across conditions, (ii) implementing a longer intervention programme than previous interventions studies and (iii) examining transference of the effects of a school-based intervention programme from a school setting to a leisure-time context.

\section{Overview of the study and hypotheses}

The present study compared effectiveness of two interventions: an autonomy-supportive intervention that provided rationale, feedback, choice and acknowledged difficulties associated with physical education classes and a less autonomysupportive intervention that provided rationale and feedback only. We decided to implement an intervention that facilitated all the components of autonomy-supportive environments because previous experimental evidence suggests that optimal performance results from environments that provide feedback, choice, rationale and acknowledge difficulties (Deci, Koestner, \& Ryan, 1999; Deci et al., 1994). Both interventions were delivered by two groups of teachers who were trained for 5 weeks to deliver the interventions. The two interventions differed in the way that feedback and rationale were communicated to pupils and in the delivery of acknowledgement of difficulties. This variation in the way that feedback and rationale (vis-á-vis acknowledgement of difficulties) was delivered allows us to test the hypothesis that autonomy-supportive teachers will be more successful in facilitating perceptions of autonomy support and autonomous motivational styles among pupils than less autonomy-supportive teachers $\left(H_{1}\right)$. Most critical, variation in the way that rationale and feedback (vis-á-vis acknowledgement of difficulties) were delivered makes our research design quite unique, in comparison to previous studies (Vansteenkiste et al., 2004b, 2005; Williams et al., 2002). This is because variation in choice and acknowledgement of difficulties allows us to test previous findings observed in laboratory settings suggesting that the effects of rationale and feedback (vis-á-vis acknowledgement of difficulties) on persistence are particularly pronounced when rationale and feedback are communicated in the context of choice (Deci et al., 1994; Ryan, 1982).

Teachers were instructed to implement the interventions for 5 weeks. After delivery of the interventions, participants were prompted to engage in leisure-time physical activities for 5 weeks and self-reported intentions to exercise during leisure time were assessed. We measured physical activity intentions in order to examine the processes by which our intervention programmes were associated with physical activity participation. 
Specifically, Deci and Ryan (1980) advocated that there are at least two ways through which cognitive elements relating to autonomy support and motivational orientations influence health behaviour. The first process, which is known as the deliberative (Fazio, 1990) or systematic (Chaiken, 1980) mode of information processing, culminates in the formation of intentions and it is associated with behaviour indirectly via intentions (Bargh, 1994; Bargh \& Chartrand, 1999). Accordingly, it was hypothesised that intentions and motivational orientations would mediate the effects of deliberative processes involving autonomy support on health behaviour $\left(H_{2}\right)$. In support of this set of assumptions, previous correlational studies showed that perceived autonomy support and motivational orientations were associated with health behaviour indirectly via intentions (Chatzisarantis, Hagger, Biddle, \& Karageorghis, 2002; Hagger et al., 2003, 2005).

The second pathway through which autonomy support and motivational orientations may predict health behaviour is via automatic processes (Deci \& Ryan, 1980). Unlike deliberative processes, which are intentional, time-consuming and effortful, automatic processes are fast, non-intentional, less effortful and a direct function of environmental cues or personal variables (Bargh, 1994). Accordingly, it was hypothesised that autonomy support and motivational orientations will display a direct relationship with health behaviour that is also independent of intentions if these variables influence health behaviour through automatic pathways $\left(\mathrm{H}_{3}\right)$. In support of this set of assumptions, previous research showed that perceptions relating to autonomy support predicted physical activity behaviour directly (Hagger et al., 2003, 2005). However, it is important to stress that in comparison to the indirect effects, the direct effects of autonomy support on health behaviour were small.

Finally, we evaluated effects of our intervention programme in the context of a larger model that took into consideration differences in the frequency participants exercised in the past through measures of past behaviour. Past behaviour effects are important to specify alongside intervention effects because effects from past behaviour can reflect effects from psychological factors that have been associated with heath behaviour in the past (Ajzen, 2002). Therefore, a conservative test of the effects of our intervention is afforded if it is shown that the intervention is associated with intentions and self-reported behaviour even after effects of past behaviour are statistically controlled (Hagger, Chatzisarantis, \& Biddle, 2002).

\section{Method}

\section{Participants}

The intervention targeted 10 co-educational schools in the South West of England. Pupils aged between 14-16 years old. Public schools, grammar schools or single-gender schools were not targeted by the intervention. Our intervention programme was delivered by five male and five female physical education teachers $(26<$ age $<28)$ who implemented changes in their teaching style for a 5-week period of time (Williams et al., 2002). This procedure resulted in the targeting of 235 pupils (Male $=106$, Female $=109$, Age $=14.84$, $\mathrm{SD}=0.40$ ). Because our research design assessed psychological variables at three points in time, there was some attrition in follow-up responses of 20 participants that resulted in a final sample of 215 pupils who successfully completed all three waves of data collection $($ Male $=106$, Female $=109$, Age $=14.84, \mathrm{SD}=0.48$, response attrition $=8.50 \%)$. It is important to stress here that 'pupils' and not 'teachers' or 'schools' were the unit of 
analysis because our intervention programme and psychological measures targeted pupils' perceptions of autonomy support and motivation.

\section{Procedure and manipulations}

We employed a simple cluster randomised design with the 10 schools being assigned, by simple random allocation, to a control or a treatment condition (Campbell, Elbourne, \& Altman, 2004). A restriction of the randomisation procedure was that an equal number of schools had to be allocated to the experimental and control conditions. In addition, to ensure that the treatment and the control conditions were not contaminated, participants from the same school were not assigned to different conditions. Five teachers in the treatment condition were instructed to adopt an autonomous inter-personal style during physical education classes. Five other teachers, the teachers of pupils in the control condition, were instructed to implement changes in their teaching style but these changes facilitated a less autonomous inter-personal style. Teachers and classes were not selected randomly but on the basis of negotiation with the school and the teachers. However, to reduce selection bias, selection of teachers and classes was made prior to the randomisation of schools in conditions and by a person masked to the purpose of the intervention.

The manipulation of pupils' perceptions of autonomy support lasted 5 weeks whereas the whole intervention study including its evaluation lasted 10 weeks. Manipulations and measurement of variables were made at three points in time and in groups of $<20$ pupils. At baseline, pupils in all conditions completed measures of past physical activity behaviour in leisure time, teacher autonomy support and measures of motivational orientations in physical education before delivery of the intervention. At follow-up 1, we measured again pupils' perceptions about teacher's autonomy support and pupils' motivational orientations in order to examine whether teachers were successful in changing pupils' perceptions of autonomy support and their motivational styles. In addition, we measured intentions to exercise during leisure time. Intentions were measured after asking pupils to exercise for at least $40 \mathrm{~min}, 4$ days per week the next 5 weeks during their leisure time at follow-up 1. At follow-up 2, self-reported physical activity behaviour was measured by using Godin and Shephard's (1985) Leisure Time Exercise Questionnaire (LTEQ).

\section{Manipulation of teacher's inter-personal style}

At baseline, the five teachers in the treatment condition were trained to adopt an autonomy-supportive inter-personal style by being instructed to provide positive feedback, rationale and acknowledge difficulties associated with physical education classes as well as enhance sense of choice by using neutral modal operators when communicating rationale, positive feedback and when acknowledging difficulties (Deci et al., 1994). Rationale was provided by instructing teachers to use a list of meaningful arguments endorsing the health benefits and mood-enhancing effects of physical exercise. Feedback was provided by instructing teachers to provide feedback related to pupils' effort rather than performance, and avoid administering controlling positive feedback (e.g. avoid saying: 'you did well, as you were supposed to'). Acknowledgement of difficulties was facilitated by instructing teachers to be empathetic and acknowledge difficulties that their pupils may experience with respect to performance of certain physical education tasks (Deci et al., 1994). Most critically, sense of choice was enhanced by instructing teachers to use neutral language 
(e.g. physical education may be fun) and not controlling modal operators (e.g. physical education should be fun) in communicating rationale feedback and in acknowledging personal difficulties. We decided to implement an intervention that facilitated all the components of autonomy-supportive environments because previous experimental evidence suggests that optimal performance results from complete autonomy-supportive environments (Deci et al., 1994; Deci et al., 1999).

Teachers in the control condition were instructed to provide rationale by using the same list of meaningful arguments that teachers in the treatment condition were instructed to use. However, these teachers were not instructed to enhance sense of choice by using neutral modal operators. Similarly, teachers in the control condition were instructed to use positive feedback without been given any special instruction about how to communicate feedback in the context of choice. Finally, the teachers in the control condition were not instructed to be empathetic and acknowledge difficulties associated with performance. Therefore, the active component of our intervention programme is concerned with provision of choice vis-á-vis acknowledgement of difficulties because provision of choice and acknowledgement of difficulties varied consistently across conditions. We decided to vary choice and acknowledgement of difficulties across conditions because previous experiential studies suggested that these are the critical factors facilitating autonomous forms of motivation and persistence (Deci et al., 1994). ${ }^{1}$

Teachers in all conditions implemented changes in their teaching style for 5 weeks. To ensure that physical education teachers understood instructions correctly, all 10 physical education teachers received the same amount of training (3 days training with $3 \mathrm{~h}$ per session) during which they had the opportunity to roleplay the recommended teaching styles and were given feedback (Williams et al., 2002). To also ensure that physical education teachers followed instructions correctly, an expert in self-determination theory observed physical education teachers in action and provided feedback about their performance.

\section{Measures}

The teacher autonomy support scale comprised 15 items, and it was adapted from the Learning Climate Questionnaire (Williams, Saizow, Ross, Deci 1997). The items yielded a score on a seven-point scale that indicated the degree to which pupils perceived that their teachers provided choice, conveyed confidence and understood their feelings and perspectives. An example item was: 'I feel that my physical education teacher has provided me choices and options'. Pupils responded to seven-point Likert scales anchored from 'strongly disagree' (1) to 'strongly agree' (7). The teacher autonomy support scale exhibited satisfactory levels of internal consistency $(\alpha=0.89)$.

Motivational orientations for physical education were measured through an approach introduced by Ryan and Connell (1989), and used in previous studies of physical education (Goudas, Biddle, \& Fox, 1994). Pupils were presented with a stem: 'I participate in physical education classes' followed by items representing an autonomous motivational style (identified regulation or intrinsic motivation) or a controlling motivational style (external regulation or introjection). An example of identification was: 'because I think physical education is important'. An example of intrinsic motivation was: 'because physical education is interesting'. An example, of external regulation was: 'because that is the rule'. An example of introjection was: 'because I will be ashamed of myself if I do not'. For the autonomous motivational orientations, the internal consistency reliabilities at baseline 
$(\alpha=0.75)$ and at follow-up $1(\alpha=0.77)$ were satisfactory. For the controlling motivational orientations, the internal consistency reliabilities were also satisfactory at baseline $(\alpha=0.85)$ and at follow-up $1(\alpha=0.76)$.

In accordance with previous research (Goudas et al., 1994; Ryan \& Connell, 1989), we calculated a relative autonomy index to represent the extent to which pupils' motivational style in physical education was autonomous versus controlling by adding the following products: external regulation $\times(-2)+$ introjection $\times(-1)+$ identification + intrinsic motivation $\times(2)$. Higher scores of this index represented a more autonomous motivational style whereas lower scores of this index represented a more controlling motivational style. At baseline, we also assessed past behaviour on a single item using a six-point scale, ranging from 'not at all' (1) to 'most of the days per week' (6) (Hagger et al., 2002). Research participants were asked to report how often they had been doing active sports, and/or vigorous physical activities for at least $40 \mathrm{~min}$, during their leisure time, the last 6 months.

Three items drawn from Ajzen and Fishbein (1980) were used to measure behavioural intentions at follow-up 1. An example was: 'I intend to do active sports and/or vigorous physical activities, for at least $40 \mathrm{~min}, 4$ days per week, during my leisure time, over the next 5 weeks', anchored by 'strongly disagree' (1) and 'strongly agree' (7). Internal consistency for the intention scale was satisfactory $(\alpha=0.93)$. At follow-up 2, we used Godin and Shephard's (1985) LTEQ to assess leisure-time physical activity. The instrument contains three open-ended questions capturing the frequency of mild, moderate and vigorous physical activity. Because the present study targeted self-reported vigorous physical activity only, participants were asked to report the frequency with which they had engaged in active sports and/or vigorous physical activities, for at least $40 \mathrm{~min}$, during leisure time, over the past 5 weeks.

\section{Data analytic strategies}

Our data analytic strategy consisted of two parts: a preliminary analysis and a main analysis. In the preliminary analysis, we conducted a 2 (intervention: yes, no) $\times$ 10 (schools) analysis of variance to examine whether participants allocated at the intervention versus control groups, and participants who were recruited from the 10 schools had similar age. In addition, in our preliminary analysis, we utilised an one-way multivariate analysis of variance to examine whether participants who completed all three waves of data collection and participants who did not complete all three waves of data collection differed on age, past behaviour, baseline motivational orientations and baseline perceived autonomy support. Further, our preliminary analysis employed an one-way multivariate analysis of variance to examine whether participants who were allocated in the intervention and control conditions differed on baseline measures of motivational orientations, perceived autonomy support and past behaviour. Most critical, our preliminary analysis also employed a 2 (intervention: yes, no) $\times 2$ (time: baseline, follow-up) repeated measures analysis of variance to examine whether participants who received the intervention displayed enhanced perceptions of autonomy support, autonomous motivational styles and enhanced levels of physical activity participation over time relative to participants who received the less autonomy-supportive intervention. Moreover, we employed a $t$-test to examine whether participants who were allocated in the intervention and control conditions differed on intentions. Finally, we calculated Person's correlations to examine relationships between variables. 
The main analysis addressed study hypotheses by utilising path analysis of observed variables (Mulaik \& Millsap, 2000). We tested hypotheses by comparing four competing models. Specifically, we initially specified a model (Model 1) that estimated covariance stabilities of physical activity behaviour, perceptions of autonomy support and motivational orientations (represented through the relative autonomy index). These covariance stabilities are represented as paths pointing from constructs measured at baseline to the same constructs measured at follow-up 1 or follow-up 2 (e.g. paths from baseline relative autonomy index to relative autonomy index measured at follow-up 1). Covariance stabilities indicate the extent to which individual differences in perceptions of autonomy support, motivational orientations and behaviour have changed over time (Hertzog \& Nesselroade, 1987). Model 1 also specified direct effects of the intervention programme on perceived autonomy support (follow-up 1) and motivational orientations (follow-up 1) (Cole \& Maxwell, 2003; Hertzog \& Nesselroade, 1987; Williams et al., 2002). These direct effects test the hypothesis that autonomy-supportive teachers will be more successful in facilitating perceptions of autonomy support and autonomous motivational styles among pupils than less autonomy-supportive teachers $\left(H_{1}\right)$. The second part of the model specified (i) indirect effects of the intervention programme on self-reported physical activity behaviour via motivational orientations (follow-up 1) and via pupils' perceptions of autonomy support (follow-up 1) and (ii) indirect effects of motivational orientations on self-reported behaviour via intentions. Moreover, we specified direct effects of past behaviour on intentions and self-reported behaviour in order to control for baseline influences of behaviour. Next, we specified a second model (Model 2) that was identical to Model 1 except of specifying direct effects from perceived autonomy support to self-reported physical activity behaviour. This direct effect was deemed necessary to be specified because previous research showed perceptions of autonomy support exhibited a direct relationship with physical activity behaviour, albeit weak (Hagger et al., 2003).

We tested our second hypothesis concerning mediating effects of autonomy support and motivational orientations on intentions and behaviour by comparing Model 2 to a competing Model 3 that tested the alternative hypothesis that autonomy support and motivational orientation did not exhibit mediating effects on intentions and behaviour. Model 3 allowed test of this alternative hypothesis because its structure was identical to the structure of Model 2 except of (i) specifying direct effects from the intervention programme on self-reported behaviour and (ii) eliminating the direct effects of the intervention programme on perceived autonomy support and motivational orientations. Similarly, we examined our third hypothesis concerning indirect effects of motivational orientations on self-reported behaviour via intentions by comparing Model 2 to a Model 4, which tested the alternative hypothesis that motivational orientations did not exhibit indirect effects on behaviour via intentions. Model 4 permitted test of this hypothesis because its structure was identical to the structure of Model 2 except of (i) specifying direct effects from motivational orientations to behaviour and (ii) eliminating the relationship between motivational orientations and intentions. Our second and third hypotheses are supported if Model 2 explains observations more satisfactorily than Models 3 and 4 and the indirect effects exhibited by the intervention programme and by motivational orientations are statistically significant (Shrout \& Bolger, 2002).

We used a polyserial matrix and Maximum Likelihood (ML) methodology to estimate parameters of the model. A polyserial matrix was the input matrix because we represented effects of the 'intervention' through a categorical variable that indicated membership in the control versus treatment conditions. When using ML methodology, it is recommended that 
a bare minimum of five participants per parameter is required, although some researchers recommended a bare minimum of 10 participants per parameter (Bentler, 1989). The estimation of our models complied with these recommendations because the most complex model (Model 2) comprised 21 parameters and consequently its estimation required a minimum of 210 participants. Further, following Roth and Switzer (1995) recommendations, we replaced missing values by using regression techniques that did not add error terms in the regression equation (Bentler, 1989).

We utilised Comparative Fit Index (CFI) and Standardised Root Mean Square Residual (SRMSR) as a means of evaluating the fit of the models because previous research has shown that these fit indices displayed restricted random variation under various conditions of model misspecification, sample size and estimation methods (Fan, Thompson, \& Wang, 1999). A cut-off value close to 0.95 for the CFI and a cut-off value close to 0.08 for the SRMSR were used to evaluate the adequacy of the ML models because the Type I and II error rates associated with these criteria are low (Hu \& Bentler, 1999). Akaike's Information Criterion (AIC) (Akaike, 1987) and the chi-square difference test $\left(\Delta x^{2}\right)$ were used to compare the competing hypothesised models (Rigdon, 1999). The model that obtains the lowest AIC is considered to demonstrate the best fit (Rigdon, 1999).

\section{Results}

\section{Preliminary analysis}

An analysis of variance did not reveal effects of the intervention, $F(1,195)=3.24, p>0.05$, $\eta^{2}=0.02$, and of schools, $F(9,195)=0.91, p>0.05, \eta^{2}=0.04$, on age. These results therefore corroborate the view that participants who were recruited from different schools and participants who were allocated to different conditions had similar age. In addition, pupils who responded at all three waves of data collection did not differ from those who were not on age, baseline measures of behaviour, baseline measures of motivational orientations, or baseline perceived autonomy support (all $F \mathrm{~s}<1$ ).

An analysis of variance using intervention as an independent variable and baseline measures of autonomy support, motivational orientations and past behaviour as dependent variables revealed a statistically significant multivariate effect of the intervention programme, $F(3,211)=15.03, p<0.05, \eta^{2}=0.18$, on motivational orientations, $F(1,213)=40.50, p<0.05, \eta^{2}=0.16$, but not on autonomy support, $F(1,213)=3.14$, $p>0.05, \eta^{2}=0.02$, or past behaviour, $F(1,213)=0.78, p>0.05, \eta^{2}=0.00$. Post hoc analysis revealed that participants in the treatment condition reported a more autonomous motivational style than participants in the control condition at baseline, $t(213)=3.41$, $p<0.05, d=0.43$. These results corroborate the view that participants assigned to different conditions differed in baseline measures of motivational orientations but not in baseline measures of behaviour or perceived autonomy support (Table 1). However, it is important to note that the effect of treatment on autonomy support was significant at $0.07 \alpha$ level. The reason for these baseline differences is that randomisation by schools has little control over the individuals (Campbell et al., 2004). Because of this variation of motivational orientations across conditions, we statistically control for baseline measures of motivational orientations and perceived autonomy support in analysis of variance and path analysis.

A repeated measures analysis of variance using the intervention as an independent variable and repeated measures of autonomy support, motivational orientations and 


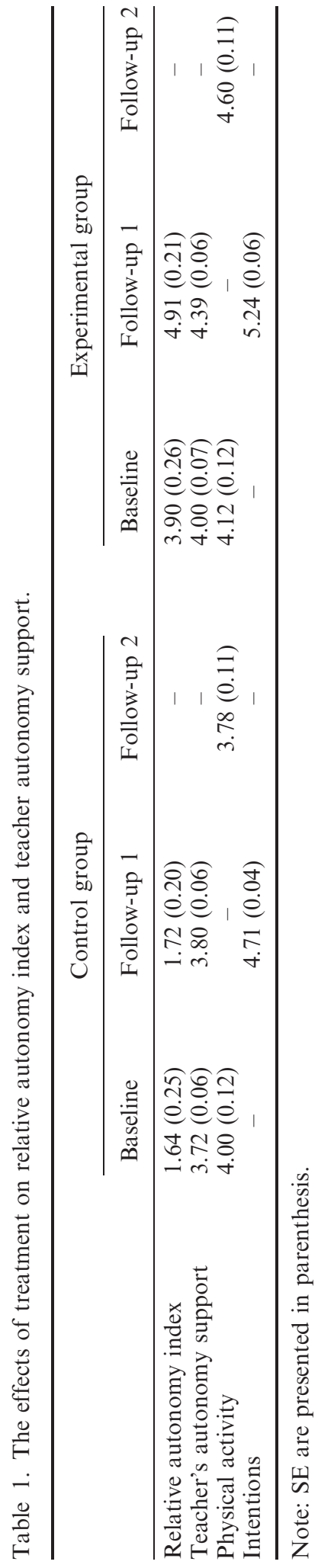


physical activity behaviour as dependent variables revealed a statistically significant interaction between treatment and time, $F(3,211)=19.75, p<0.05, \eta^{2}=0.22$, on repeated measures of teacher autonomy support, $F(1,213)=80.33, p<0.05, \eta^{2}=0.18$, on repeated measures of motivational orientations, $F(1,213)=19.34, p<0.05, \eta^{2}=0.11$ and on repeated measures of self-reported physical activity behaviour, $F(1,213)=20.70, p<0.05$, $\eta^{2}=0.09$. The effect of time on dependent variables was also statistically significant, $F(3,211)=67.02, p<0.05, \eta^{2}=0.19$. Post hoc analysis revealed that while pupils in the treatment condition exercised more frequently, $F(1,101)=24.48, p<0.05, \eta^{2}=0.18$, perceived their teachers as more autonomy supportive, $F(1,101)=61.40, p<0.05$, $\eta^{2}=0.34$, and reported a more autonomous motivational orientation, $F(1,101)=22.40$, $p<0.05, \eta^{2}=0.20$, at follow-up 1 than at baseline, this was not necessarily the case for pupils in the control condition (Table 1). Pupils in the control condition did not report enhanced autonomous motivational orientation, $F(1,112)=2.34, p>0.05, \eta^{2}=0.02$, as well as they exercised less, $F(1,112)=3.85, p<0.05, \eta^{2}=0.03$, and they perceived their teacher as being less autonomy supportive over time, $F(1,112)=36.77, p<0.05, \eta^{2}=0.25$. Finally, a $t$-test revealed a statistically significant effect of treatment on intentions, $t(213)=5.60, p<0.05, d=0.73$. In accordance with initial hypothesis, post hoc analysis revealed that participants in the treatment condition reported stronger intentions to exercise $(\mathrm{M}=5.21, \mathrm{SE}=0.11)$ than participants in the control condition $(\mathrm{M}=4.26$, $\mathrm{SE}=0.12)$.

Table 2 presents correlations between psychological variables. As shown, the intervention programme was positively associated with pupils' perceptions of autonomy support (baseline and follow-up 1), pupils' reports of autonomous motivation (baseline and follow-up 1), intentions and self-reported physical activity participation. In addition, pupils' perceptions of autonomy support and autonomous motivation assessed at followup 1 were positively associated with self-reported physical activity participation and intentions. Moreover, intentions were positively associated with self-reported physical activity participation (Table 2). Finally, past behaviour was positively associated with intentions, self-reported physical activity behaviour, perceptions of autonomy support (baseline and follow-up 1) and autonomous motivation (baseline and follow-up 1). The correlation between past and future behaviour was large and comparable with correlations reported in previous studies investigating physical activity (Hagger et al., 2002).

\section{Path analysis}

Path analysis indicated that only Model 2, the model that hypothesised direct effects of perceived autonomy support on self-reported physical activity, exceeded recent criteria of good fit. Model 1, the model that did not hypothesise direct effects of perceived autonomy support on self-reported physical activity, did not exceed recent criteria of good fit (see Table 3). In accordance with our second hypothesis, Model 2 displayed better fit than Model 3, the model that hypothesised direct and not indirect effects of the intervention programme on self-reported physical activity, $\Delta x^{2}(1, N=208)=131.87, p<0.05$. Similarly, in support of our third hypothesis, Model 2 displayed better fit than the Model 4, the model that hypothesised direct and not indirect effects of motivational orientations on self-reported physical activity (see Table 3).

Parameters of Model 2 lent further support to our initial hypotheses. Specifically, in accordance with hypothesis 1, parameters of the model revealed that the intervention programme was successful in enhancing perceptions of autonomy support and 


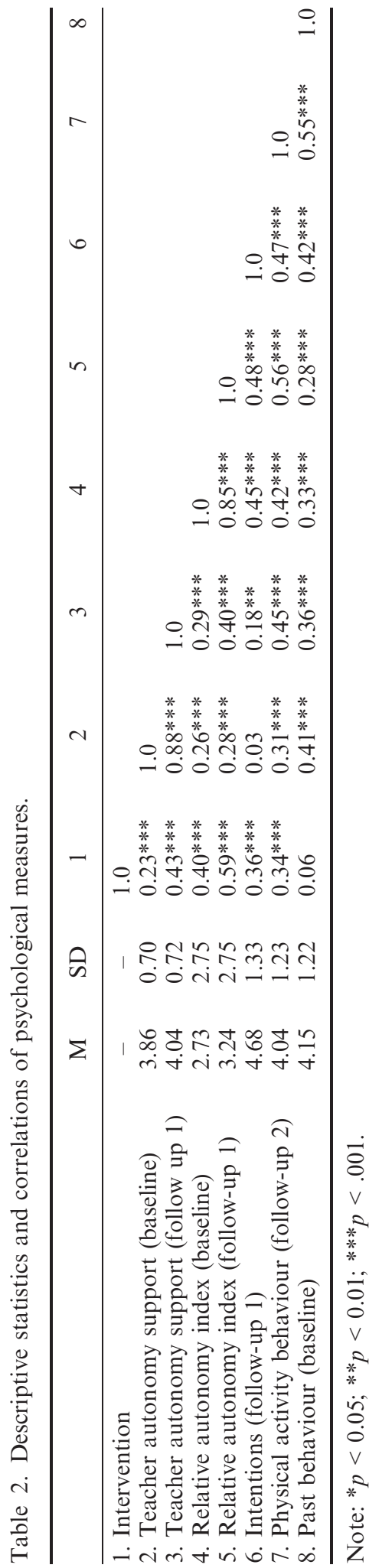


Table 3. Fit indexes of hypothesised models.

\begin{tabular}{lrccr}
\hline & \multicolumn{1}{c}{$X^{2}(d f)$} & CFI & SRMSR & AIC \\
\hline Model 1 & $104.93(12)$ & 0.92 & 0.06 & 82.93 \\
Model 2 & $65.68(11)$ & 0.95 & 0.06 & 43.68 \\
Model 3 & $215.62(12)$ & 0.83 & 0.11 & 191.62 \\
Model 4 & $205.22(12)$ & 0.84 & 0.12 & 181.22 \\
\hline
\end{tabular}

Note: The CFI for Models 1, 2, 3 and 4 exceeded 0.90 when using generalised least square methodology.

autonomous motivational styles given that the direct effects of the intervention on autonomous motivation (follow-up 1) and perceptions of autonomy support (follow-up 1) were positive and statistically significant (Figure 1). In addition, consistent with our hypothesis 2, Model 2 revealed positive indirect effects of the intervention programme on self-reported physical activity behaviour via autonomous motivation (follow-up 2) and intentions $(\beta=0.06, p<0.05)$. Sobel (1982) tests also revealed that the indirect effects of the intervention programme on intentions via autonomous motivation (follow-up 1) were also statistically significant $(\beta=0.11, z=2.56, p<0.05)$. Interestingly, the model explained over $64 \%$ of variance on self-reported physical activity behaviour $\left(f^{2}=2.88\right)$. In accordance with previous research (Hagger et al., 2003, 2005), perceived autonomy support exerted a positive direct effect on self-reported behaviour but its indirect effect on intentions and self-reported physical activity via motivational orientations was not statistically significant. Moreover, consistent with hypothesis 3, Sobel (1982) tests revealed positive indirect effects of motivational orientations on self-reported physical activity behaviour via intentions $(\beta=0.20, z=3.27, p<0.05)$. The direct effects of motivational orientations on self-reported physical activity behaviour were not statistically significant (Figure 1). Finally, in accordance with previous research (Hagger et al., 2002), past behaviour exerted positive direct effects on intentions and self-reported physical activity behaviour. $^{2}$

\section{Discussion}

The purpose of the present study was to evaluate utility of an intervention programme based on self-determination theory in changing perceptions of autonomy support, motivational orientations, intentions and self-reported physical activity participation. In accordance with hypothesis 1 , the multivariate analysis of variance and the path analysis indicated that pupils perceived teachers who adopted an autonomy-supportive interpersonal style as being more autonomy supportive and reported a more autonomous motivational orientation from baseline to follow-up 1. In contrast, in the control condition, pupils' perceptions related to autonomy support and motivational styles did not change over time. These results are consistent with tenets of self-determination theory and previous research (Hagger et al., 2003; Reeve \& Jang, 2006; Ryan \& Deci, 2000), and suggest that our 5-week intervention programme was successful in enhancing pupils' perceptions of autonomy support and autonomous motivational orientation. The more the teachers were autonomy supportive during physical education classes, the more the pupils perceived their teachers to provide choice and to be meaningfully related to them. In addition, pupils who were taught by autonomy-supportive teachers were more likely to report autonomous motivational styles and therefore report that physical education was 


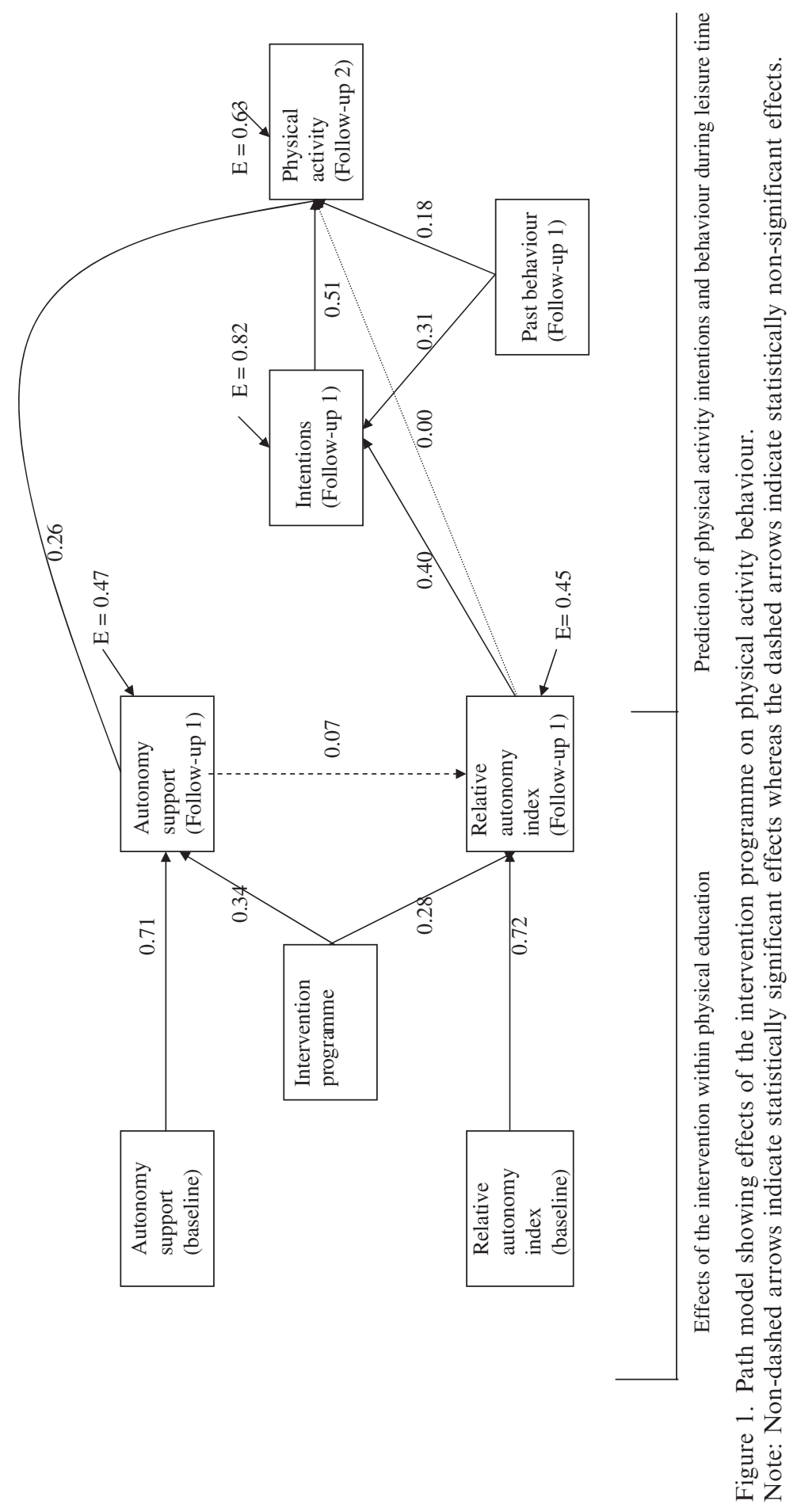


important and enjoyable subject whereas this was not the case for pupils in the control condition.

The fact that pupils in the intervention condition reported enhanced perceptions of autonomy support is consistent with tenets of self-determination theory concerning the nature of autonomous inter-personal styles and environments. Specifically, Deci and Ryan (1985) suggested that the provision of meaningful rationale and feedback impacts on people's perceptions of autonomy support and motivational orientations only when rationale and feedback are communicated in the context of autonomy (Deci et al., 1994; Vansteenkiste et al., 2004b, 2005). Sense of autonomy can be enhanced by using neutral language during inter-personal contact (e.g. use of 'mays', 'coulds' and not 'shoulds' and 'musts'). In accordance with this proposition, results of the present study indicated that pupils reported enhanced perceptions of autonomy support when they were taught by teachers who communicated rationale and feedback autonomously vis-á-vis acknowledged personal feelings and perspectives. Pupils did not change their perceptions of autonomy support and perceived motivational orientation when acknowledgement of personal feelings and perspectives did not take place and when rationale and feedback were not communicated in the context of autonomy.

Another important finding raised by the present study is concerned with the effects of the intervention programme on self-reported physical activity participation. In partial support of hypothesis 2, the multivariate analysis of variance and the path analysis demonstrated that our school-based intervention programme that targeted physical education increased the frequency with which young people engaged in physical activities during leisure time by promoting a more autonomous motivational style during physical education. These results compare favourably with basic tenets of self-determination theory that posit that environmental factors such as rewards, feedback and inter-personal variables influence behaviour indirectly via motivational orientations (Deci \& Ryan, 1980, 1985). That is, according to this perspective, environmental factors promote performance of health behaviours only when they facilitate an autonomous motivational style (Deci \& Ryan, 1985). However, the path analysis did not support similar effects of perceived autonomy support on self-reported behaviour via motivational orientations, despite that correlations supported a positive relationship between perceived autonomy support and motivational orientations (Table 1). The absence of an effect for perceived autonomy support may reflect a general limitation of measures of perceived autonomy support to capture all aspects of our intervention. For instance, the measure of perceived autonomy support assessed perceived provision of feedback through one item only, yet our intervention programmes instructed teachers to consistently provide feedback throughout the intervention period. This inadequate measurement of certain aspects of autonomy support might have reduced the utility of our measures of perceived autonomy support to completely mediate effects of the intervention programme on motivational orientations by allowing aspects of our intervention programme to exert direct effects on motivational orientation and consequently attenuate the effects of perceived autonomy support on intentions.

Not only did the present study replicate previous important findings but it also extended previous research in several ways. In particular, the present study is one of few interventions that targeted leisure-time physical activity participation and supported utility of autonomy-supportive interventions in facilitating transference of exercise behaviour from a school setting to a leisure context (Vansteenkiste et al., 2004b, 2005; Williams et al., 2002). Our intervention supports transference of behaviour because the indirect effects of our intervention programme on behaviour via motivational orientations support the 
notion that promotion of autonomous motivational orientation during physical education, brought by our autonomy-supportive physical education programmes, increased the frequency with which young people engaged in physical activity during leisure time. Most important, our study provides a rigorous test of hypothesis related to transference of behaviour from one context to another. This is because our study did not provide young people with an exercise programme and a setting where they could exercise after termination of the intervention, during leisure time. Rather, our study required from participants to organise and plan their leisure-time physical activity participation, a requirement that is clearly much more demanding than the requirement to participate in a specific exercise programme (Vansteenkiste et al., 2004b).

In addition, in comparison to previous research, the effects observed for our intervention on self-reported health behaviour were large and independent of past physical activity behaviour. Indeed, the indirect effects of our intervention programme on self-reported physical activity participation via motivational orientations were $0.06 ; 6$ times larger than in Williams et al. (2002) study. Moreover, while in Vansteenkiste et al. (2004b), the size of the effects $\left(f^{2}\right)$ of autonomy support on sport participation ranged from 0.02 to 0.27 , in our intervention programme the effect size explaining self-reported physical activity participation was 0.85 . One reason for this is that Williams et al.'s and Vansteenkiste et al.'s intervention programmes were brief. In contrast, our intervention comprised long physical education classes $(45 \mathrm{~min})$, which were spread over a 5-week interval of time. In addition, unlike previous studies that used a controlling inter-personal style as a contrasting condition (Vansteenkiste et al., 2004b, 2005; Williams et al., 2002), the present study adopted more stringent criteria and demonstrated effects of an intervention programme over and above a control condition in which participants were provided with a rationale and feedback but not in an autonomous or controlling way. Our control group provides a more stringent test of intervention effects because controlling inter-personal styles result in lower levels of motivation than interventions that provide positive feedback and rationale (Deci \& Ryan, 1985; Deci et al., 1994).

The present study not only demonstrates the effects of an intervention programme based on self-determination theory on self-reported health behaviour, but it also incorporates measures of intentions in an attempt to ascertain the processes by which autonomous inter-personal styles and motivational orientations were associated with health behaviour. In accordance with hypothesis 3, the path analysis indicated that our intervention programme and the autonomous motivational styles adopted by young people during physical education brought changes on self-reported physical activity participation by changing young people's intentions to exercise during leisure time. These results compare favourably with previous physical activity research (Hagger et al., 2003, 2005) and corroborate the view that the processes by which autonomous motivational styles increased self-reported physical activity participation were deliberative and not automatic (Bargh, 1994; Fazio, 1990). These deliberative processes also imply that young people whose motivation is autonomous during physical education classes are more likely to exercise during leisure time than young people whose motivation is not autonomous during physical education perhaps because autonomous experiences associated with physical education instigate general tendencies to plan participation in leisure-time physical activities.

One reason for observing deliberative processes only is that the nature of our intervention programme encouraged deliberative process (planning) rather than an automatic process. According to Fazio (1990), individuals engage in automatic processing when the circumstances call for a fast decision to be made, when information relating to 
the pros and cons of the behaviour is scarce and is perceived to originate from a credible source and when opportunities for performing the behaviour are clear. In those circumstances, people may decide to exercise simply because the information comes from a credible and trustworthy source. In contrast, when there is ample time and information for individuals to deliberate as well as individuals are motivated to deliberate the pros and cons of the behaviour then individuals may exercise because they believe that physical activity is useful and beneficial. Clearly, our intervention strategies encouraged a deliberative process of influence (planning) because (i) they prompted young people to exercise without offering them with an opportunity to do so and (ii) they provided (at follow-up 1) plenty of time for individuals to deliberate over their participation in leisuretime physical activities. In addition, although the information provided to pupils was credible in a sense that it originated from physical education teachers, it was ample given that physical education teachers were providing meaningful rationale for 5 weeks. A direct effect of motivational orientations on physical activity participation might have been observed if teachers provided limited amount of information regarding the benefits of physical exercise (Fazio, 1990). Future experimental research should investigate whether autonomous motivational styles can influence health behaviour through automatic pathways by presenting pupils with credible information for a limited time (Bargh \& Chartrand, 2000).

A final finding that deserves discussion is concerned with the direct relationships between perceptions of autonomy support and self-reported physical activity participation. Specifically, the path analysis documented that perceptions of autonomy support measured at follow-up 1 exerted strong direct effects on self-reported physical activity participation (Figure 1). This direct effect may due to the fact that the model tested in the present study does not take into consideration experiences relating to need satisfaction (e.g. experiences of competence, autonomy and relatedness). Indeed, previous studies observed direct effects of perceived autonomy support on self-reported physical activity participation only when experiences related to need satisfaction were not incorporated in the model (Hagger et al., 2003, 2005) and not when experiences related to need satisfaction were taken into consideration (Ntoumanis, 2005; Standage et al., 2005). Future experimental studies should attempt to replicate results of our study and extend them by incorporating measures relating to need satisfaction. The effects of need satisfaction on behaviour may be both direct reflecting automatic processes of influence and indirect (via intentions) reflecting influences due to deliberative processes.

Although our study demonstrated effects of a long intervention programme on physical activity intentions, it is not without limitations. One obvious limitation has to do with the scale of the intervention. Specifically, our intervention programme was applied to a limited number of comprehensive schools and pupils and the sample size of our study might have been small. Therefore, results of the present study may not generalise to other types of schools or to pupils with different learning and physical abilities. Future studies may attempt to replicate results of the present study by conducting a larger scale intervention. In addition, as stated previously, our model may omit important variables such as variables reflecting satisfaction of psychological needs. Therefore, future studies may attempt to re-examine whether experiences related to satisfaction of the needs for competence, autonomy and relatedness further mediate the effects of perceptions of autonomy support on intentions and behaviour. Further, our intervention involved the manipulation of several components of autonomy support such as feedback, sense of autonomy and acknowledgement of difficulties. Therefore, future work might examine 
whether all or only one of the manipulated components yield independent effects on motivational orientations.

Moreover, it is important to note that our study targeted pupils' perceptions of autonomy support and not pupils' goals. Recent developments indicated that in addition to perceived autonomy support, the type of the goals people pursue (whether the goals are extrinsic or intrinsic) exerted large effects on health behaviour (Vansteenskiste et al., 2004a, 2005). Indeed, Vansteenkiste et al. (2004) reported effect sizes $\left(f^{2}\right)$ for the effect of autonomy support and intrinsic goals on sport participation ranging from 0.17 to 0.87. Future, studies should examine whether long-term intervention programmes that target perceptions of autonomy support and intrinsic goals exert stronger effects on physical activity participation. What is more, although our control group provided a stringent test of the intervention programme, it overlapped with the content of the intervention given that rationale and feedback are components of autonomy-supportive environments. Therefore, future studies may adopt more complete designs that compare intervention effects against a control group in which participants are not provided with rationale and feedback but they are simply asked to engage in leisure-time physical activity. In addition, future studies may manipulate several components of autonomy support to examine whether all or only one of the manipulated components produce a significant effect. Finally, our self-reported measure of physical activity behaviour refers to a relatively long period and therefore responses may be distorted due to memory bias (Stone \& Shiffman, 2002). Future, research may replicate findings of the present study against more objective measures of physical activity behaviour.

In conclusion, the present study extends current knowledge by utilising a long-term intervention programme to support the motivational sequence proposed by selfdetermination theory. Results confirm that teacher's inter-personal orientation changes self-reported physical activity participation during leisure time via the mediation of pupils' perceptions of autonomy support, reports of autonomous motivation, and intentions. The implications of these findings is that tenets of self-determination theory can be embedded in existing educational curricular and help physical education teachers achieve targets related to the promotion of leisure-time physical activity.

\section{Notes}

1. Full details of the intervention are available from the corresponding author.

2. We also re-analysed the data by splitting the autonomy support scale into three dimensions: support of competence, support of relatedness and support of autonomy. Results from this analysis revealed that the intervention was more likely to improve perceptions related to competence support, $F(1,213)=27.91, p<0.05$, autonomy support, $F(1,213)=31.37, p<0.05$ and relatedness support, $F(1,213)=26.28, p<0.05)$ among participants who received the intervention than among individuals in the control group. In addition, regression analysis revealed small indirect effects of the intervention programme on motivational orientations via perceived competence support, perceived relatedness support and perceived autonomy support ( $\beta$-coefficients reflecting indirect effects were below 0.05 ).

\section{References}

Ajzen, I. (2002). Residual effects of past on later behavior: Habituation and reasoned action perspectives. Personality and Social Psychology Review, 6(2), 107-122. 
Ajzen, I., \& Fishbein, M. (1980). Understanding attitudes and predicting social behavior. Englewood Cliffs, NJ: Prentice-Hall.

Akaike, H. (1987). Factor analysis and AIC. Psychometrika, 52(3), 317-332.

Bargh, J.A. (1994). The Four Horsemen of automaticity: Awareness, efficiency, intention and control in social cognition. In R.S. Wyer, Jr., \& T.K. Srull (Eds.), Handbook of social cognition (2nd ed., pp. 1-40). Hillsdale, NJ: Erlbaum.

Bargh, J., \& Chartrand, J. (1999). The unbearable automaticity of being. American Psychologist, 54(7), 462-479.

Bargh, J.A., \& Chartrand, T.L. (2000). The mind in the middle: A practical guide to priming and automaticity research. In H.T. Reis, \& C.M. Judd (Eds.), Handbook of research methods in social and personality psychology (pp. 253-285). New York: Cambridge University Press.

Bentler, P.M. (1989). EQS structural equations program manual. Los Angeles: BMDP Statistical Software.

Campbell, M.K., Elbourne, D.R., \& Altman, D.G. (2004). Consort statement: Extension to cluster randomized trials. British Medical Journal, 328(7441), 702-708.

Chaiken, S. (1980). Heuristic versus systematic information processing and the use of source versus message cues in persuasion. Journal of Personality and Social Psychology, 39(5), 752-766.

Chatzisarantis, N.L.D., Hagger, M.S., Biddle, S.J.H., \& Karageorghis, C. (2002). The cognitive processes by which perceived locus of causality predicts participation in physical activity. Journal of Health Psychology, 7(6), 685-699.

Cole, D.A., \& Maxwell, S.E. (2003). Testing mediational models with longitudinal data: Questions and tips in the use of structural equation modeling. Journal of Abnormal Psychology, 112(4), 558-577.

Deci, E.L., Eghrari, H., Patrick, B.C., \& Leone, D. (1994). Facilitating internalization: The selfdetermination theory perspective. Journal of Personality, 62, 119-142.

Deci, E.L., Koestner, R., \& Ryan, R.M. (1999). A meta-analytic review of experiments examining the effects of extrinsic rewards on intrinsic motivation. Psychological Bulletin, 125, 627-668.

Deci, E.L., \& Ryan, R.M. (1980). Self-determination theory: When mind mediates behavior. The Journal of Mind and Behavior, 62(1), 33-43.

Deci, E.L., \& Ryan, R.M. (1985). Intrinsic motivation and self-determination in human behavior. New York: Plenum Publishing Co.

Fan, X., Thompson, B., \& Wang, L. (1999). Effects of sample size, estimation methods and model specification on structural equation modelling. Structural Equation Modeling: A Multidisciplinary Journal, 6(1), 56-83.

Fazio, R.H. (1990). Multiple processes by which attitudes guide behavior: The mode model as an integrative framework. In M.P. Zanna (Ed.), Advances in Experimental Social Psychology (pp. 75-109). New York: Academic Press.

Godin, G., \& Shephard, R.J. (1985). A simple method to assess exercise behavior in the community. Canadian Journal of Applied Sport Science, 10(3), 141-146.

Goudas, M., Biddle, S., \& Fox, K. (1994). Perceived locus of causality, goal orientations, and perceived competence in school physical education classes. British Journal of Educational Psychology, 64(3), 453-463.

Hagger, M., Chatzisarantis, N., \& Biddle, S. (2002). A meta-analytic review of the theories of reasoned action and planned behavior: Predictive validity and the contribution of additional variables. Journal of Sport and Exercise Psychology, 24(1), 3-32.

Hagger, M.S., Chatzisarantis, N.L.D., Barkoukis, V., Wang, C.K.J., \& Baranowski, J. (2005). Perceived autonomy support in physical education and leisure-time physical activity: A crosscultural evaluation of the trans-contextual model. Journal of Educational Psychology, 97(2), 287-301.

Hagger, M.S., Chatzisarantis, N.L.D., Culverhouse, T., \& Biddle, S.J.H. (2003). The process by which perceived autonomy support in physical education promotes leisure-time physical activity intentions and behavior: A trans-contextual model. Journal of Educational Psychology, 95(4), 784-795. 
Hertzog, C., \& Nesselroade, J.R. (1987). Beyond autoregressive models: Some implications of the trait-state distinction for the structural modelling of developmental change. Child Development, 58(1), 93-109.

Hu, L., \& Bentler, M. (1999). Cut-off criteria for fit indexes in covariance structure analysis: Conventional criteria versus new alternatives. Structural Equation Modeling: A Multidisciplinary Journal, 6(1), 1-55.

Mulaik, A., \& Millsap, R.E. (2000). Doing the four-step right. Structural Equation Modeling: A Multidisciplinary Journal, 7(1), 36-73.

Ntoumanis, N. (2001). A self-determination approach to the understanding of motivation in physical education. British Journal of Educational Psychology, 71(2), 225-242.

Ntoumanis, N. (2005). A prospective study of participation in optional school physical education using a self-determination theory framework. Journal of Educational Psychology, 97(3), 444-453.

Reeve, J., \& Jang, H. (2006). What teachers say and do to support students' autonomy during a learning activity. Journal of Educational Psychology, 98(1), 209-218.

Rigdon, E.E. (1999). Using the Friedman method of ranks for model comparison in structural equation modelling. Structural Equation Modelling: A Multidisciplinary Journal, 6(3), 219-232.

Roth, P.L., \& Switzer, F.S. (1995). A Monte-Carlo analysis of missing data techniques in HRM setting. Journal of Management, 21(5), 1003-1023.

Ryan, R.M. (1982). Control and information in the intrapersonal sphere: An extension of cognitive evaluation theory. Journal of Personality and Social Psychology, 43(3), 450-461.

Ryan, R.M., \& Connell, J.P. (1989). Perceived locus of causality and internalization: Examining reasons for acting in two domains. Journal of Personality and Social Psychology, 57(5), $749-761$.

Ryan, R.M., \& Deci, E.L. (2000). Self-determination theory and the facilitation of intrinsic motivation, social development, and well-being. American Psychologist, 55(1), 68-78.

Shrout, P.E., \& Bolger, N. (2002). Mediation in experimental and nonexperimental studies: New procedures and recommendations. Psychological Methods, 7(4), 422-445.

Sobel, M.E. (1982). Asymptotic intervals for indirect effects in structural equations models. In S. Leinhart (Ed.), Sociological methodology (pp. 290-312). San Francisco: Jossey-Bass.

Standage, M., Duda, J.L., \& Ntoumanis, N. (2003). A model of contextual motivation in physical education: Using constructs from self-determination theory and achievement goal theory to predict physical activity intentions. Journal of Educational Psychology, 95(1), 97-110.

Standage, M., Duda, J.L., \& Ntoumanis, N. (2005). A test of self-determination theory in school physical education. British Journal of Educational Psychology, 75(4), 411-433.

Stone, A.A., \& Shiffman, S. (2002). Capturing momentary, self-report data: A proposal for reporting guidelines. Annals of Behavioral Medicine, 24(2), 236-43.

Vansteenkiste, M., Simons, J., Lens, W., Sheldon, K.M., \& Deci, E.L. (2004a). Motivating learning, performance, and persistence: The synergistic effects of intrinsic goal contents and autonomysupportive contexts. Journal of Personality and Social Psychology, 87(2), 246-260.

Vansteenkiste, M., Simons, J., Lens, W., Soenens, B., \& Matos, L. (2005). Examining the motivational impact of intrinsic versus extrinsic goal framing and autonomy-supportive versus internally controlling communication style on early adolescents' academic achievement. Child Development, 76(2), 483-501.

Vansteenkiste, M., Simons, J., Soenes, B., \& Lens, W. (2004b). How to become a persevering exerciser? Promoting a clear, future intrinsic goal in an autonomy supportive way. Journal of Sport and Exercise Psychology, 2(2), 232-249.

Williams, G.C., Gagné, M., Ryan, R.M., \& Deci, E.L. (2002). Facilitating autonomous motivation for smoking cessation. Health Psychology, 21(1), 40-50.

Williams, G.C., Saizow, R., Ross, L., \& Deci, E.L. (1997). Motivation underlying career choice for internal medicine and surgery. Social Science and Medicine, 45(9), 1705-1713. 
Copyright of Psychology \& Health is the property of Routledge and its content may not be copied or emailed to multiple sites or posted to a listserv without the copyright holder's express written permission. However, users may print, download, or email articles for individual use. 